\title{
Laboratório de tratamento e recuperação de resíduos químicos: alternativa para resíduos industriais do sul de Minas Gerais
}

\author{
Luciano Tavares da Costa \\ Programa de Pós-Graduação em Química da Universidade Federal Fluminense (Uff) \\ Eduardo Gomes Salgado \\ Programa de Pós-Graduação em Ecologia e Tecnologia Ambiental da Universidade Federal de Alfenas (Unifal) \\ Dirlane de Fátima do Carmo \\ Programa de Pós Graduação em Engenharia de Biossistemas da Universidade Federal Fluminense (Uff) \\ Mayra Guerra \\ Programa de Pós-Graduação em Ecologia e Tecnologia Ambiental da Universidade Federal de Alfenas (Unifal) \\ Marina Evangelista \\ Programa de Pós-Graduação em Ecologia e Tecnologia Ambiental da Universidade Federal de Alfenas (Unifal) \\ Karla Silveira \\ Programa de Pós-Graduação em Ecologia e Tecnologia Ambiental da Universidade Federal de Alfenas (Unifal)
}

Recebido: 28/01/2015 Versão revisada (entregue): 06/05/2015 Aprovado: 08/05/2015

\begin{abstract}
Resumo
Este artigo tem como objetivo a obtenção de dados, como custos, equipamentos e investimentos necessários para a construção, na UNIFAL-MG (campus II, em Alfenas), de um laboratório de tratamento e recuperação de resíduos químicos gerados na Universidade. Para auxiliar na decisão de implantação e operação do laboratório foi utilizado o EVTE (Estudo de Viabilidade Técnica e Econômica). O trabalho teve as seguintes etapas: identificação e quantificação dos resíduos, elaboração do EVTE, esboço da planta, captação de recursos e submissão do projeto. Verificou-se que a implantação e gestão do laboratório atenderiam a um volume inicial de aproximadamente $372 \mathrm{~L} /$ mês de resíduos químicos e $3,5 \mathrm{~kg} / \mathrm{mês}$ de sais inorgânicos processados, além de outros resíduos de indústrias da região. A construção e manutenção deste laboratório são viáveis, dependendo do volume de resíduos tratados, recuperados ou reciclados, bem como da prestação de serviço para geradores de resíduos químicos da região. Deve-se ressaltar o benefício socioambiental e econômico devido à redução da quantidade (e a nocividade) do descarte de resíduos químicos, a oportunidade de formação acadêmica e a conscientização social promovida pela ação do laboratório, além de adequação aos princípios do Plano de Logística Sustentável na Administração Pública Federal.
\end{abstract}


Palavras-chave | Laboratório; Minas Gerais; reciclagem; resíduos químicos; sustentabilidade.

Código JEL | Q01; QR3; L65.

\title{
CHEMICAL WASTE TREATMENT AND RECOVERY LABORATORY: AN ALTERNATIVE FOR INDUSTRIAL WASTE OF SOUTHERN MINAS GERAIS
}

\begin{abstract}
This manuscript consisted to obtain data, such as costs, equipments and investments necessary for the implementation of a Waste Treatment and Recovery Laboratory at UNIFAL-MG, campus II in Alfenas. In order to give support for the implementation and operation of this laboratory, in a way to guarantee a sustainable investment from the economic point of view, the EVTE was applied. This work was performed following the steps: identification and quantification of the wastes, EVTE elaboration, draft of the physical laboratory architecture and the analysis of the potential financial resources. It was verified that the implementation and management of the Chemical Waste Treatment Laboratory get to support an initial waste volume of $372 \mathrm{~L} /$ month and $3.5 \mathrm{~kg} /$ month of inorganic salts, beyond other industrial wastes from the neighborhood region. The implementation and maintenance of this laboratory are economic viable depending on the treated, recovered and recycled waste volume as well as on the provided service for the industry client. It is necessary to highlight the environmental benefits, especially due to the chemical waste disposal reduction, the academic formation opportunity and the social awareness promoted by the action of the laboratory. It can be add on the principle related to the Sustainable Logistic Plan in the Federal Public Administration.
\end{abstract}

Keywords | Chemical residues; laboratory; Minas Gerais; recycling; sustainability.

JEL-Code | Q01; QR3; L65.

\section{LABORATORIO DE TRATAMIENTO Y RECUPERACIÓN DE RESIDUOS QUÍMICOS: ALTERNATIVA PARA RESIDUOS INDUSTRIALES EN EL SUR DE MINAS GERAIS}

\section{Resumen}

En este artículo se pretende obtener datos tales como los costos, el equipo y las inversiones necesarias para la construcción, en la UNIFAL-MG (campus II, en la ciudad de Alfenas), de un laboratorio de tratamiento y recuperación de residuos químicos generados en la Universidad. Para ayudar en la decisión para la ejecución y la operación del laboratorio se utilizó el EVTE (Estudio de viabilidad técnica y económica). El trabajo pasó por las siguientes etapas: identificación y cuantificación de los residuos, preparación del EVTE, elaboración de la planta, recaudación de fondos y presentación del proyecto. Se encontró que el despliegue y gestión del laboratorio se encontrarían en un volumen inicial de aproximadamente $372 \mathrm{~L} / \mathrm{m}$ de desechos químicos y $3,5 \mathrm{~kg} / \mathrm{mes}$ de sales inorgánicas procesadas, además de otros residuos de industrias de la región. La construcción y el mantenimiento de este laboratorio son factibles, dependiendo del volumen de residuos tratados, recuperados o reciclados, así como es factible la prestación de servicios para los generadores de residuos químicos de la región. Debe tenerse en cuenta el beneficio socio-ambiental y económico debido a la disminución de la cantidad (y del daño) por la eliminación de residuos químicos, la oportunidad de formación académica y la conciencia social promovida por la acción del laboratorio, además de la adecuación a los principios del Plan de Logística Sostenible en la administración pública federal. 
Palabras-clave | Laboratorio; Minas Gerais; reciclaje; residuos químicos; sostenibilidad.

Código JEL | Q01; QR3; L65.

\section{Introdução}

A preocupação com o meio ambiente e medidas visando a redução da degradação ambiental não são recentes. Nesse sentido, uma política ambiental cada vez mais integrada vem sendo instituída ao longo dos anos no Brasil, como pode ser observado na Política Nacional de Meio Ambiente (BRASIL, 1981), na Política Nacional de Recursos Hídricos (BRASIL, 1997) e na recente Política Nacional de Resíduos Sólidos (BRASIL, 2010). Além disso, a população tem demonstrado uma preocupação crescente com o meio ambiente. Como aponta Escobar (2012), uma pesquisa feita a pedido da Confederação Nacional da Indústria (CNI) revelou que aumentou o percentual de pessoas que se dizem preocupadas com o meio ambiente, tendo passado de 80\%, em 2010, para 94\%, em 2011. Deve-se ressaltar ainda que $44 \%$ dos entrevistados afirmaram que a proteção ao meio ambiente tem prioridade sobre o crescimento econômico (ESCOBAR, 2012).

Promover o desenvolvimento econômico em bases sustentáveis é um tema relevante que vem sendo discutido pelos países desde a Conferência das Nações Unidas sobre o Meio Ambiente e Desenvolvimento realizada em Estocolmo no ano de 1972, tendo sido cunhado o termo ecodesenvolvimento, que passou a desenvolvimento sustentável na conferência realizada no Rio de Janeiro em 1992.

De acordo com Hanai (2011), o termo "desenvolvimento sustentável" é considerado por muitos como uma utopia, sendo um modelo abstrato e imaginário da sociedade atual. Para ele, o desenvolvimento refere-se à necessidade de mudar a qualidade do crescimento. Porém, apesar da ambiguidade, o desenvolvimento sustentável tem envolvido um conjunto central de princípios e valores orientadores.

Isto porque a ideia de desenvolvimento sustentável surge na busca do equilíbrio entre o crescimento econômico, a preservação do meio ambiente e a resolução das desigualdades sociais. Para se por em prática a sustentabilidade é imprescindível a utilização de algumas ferramentas para guiar as ações e avaliar se há progresso rumo a esta. A efetividade das ferramentas utilizadas pode ser avaliada por meio de indicadores que servem para agregar e quantificar informações de um conjunto de dimensões que se pretende avaliar. Algumas metodologias de análise de sistemas de indicadores são o Painel de Sustentabilidade (IISD, 2007), Índice de Sustentabilidade Ambiental (ESTY et al., 2005) e o Barômetro de Sustentabilidade (PRESCOTT-ALLEN, 2001), entre outros. 
A sustentabilidade incentiva o desenvolvimento integrado, com a promoção do crescimento da igualdade de condições para os indivíduos (LUCENA, CAVALCANTI; CÂNDIDO, 2010). Busca-se, assim, a transformação do crescimento econômico capitalista em um modelo que atenda as necessidades das gerações atuais, sem comprometer a qualidade de gerações futuras, como preconizado no relatório da Comissão Mundial para o Meio Ambiente e Desenvolvimento (CMMAD). Este relatório, conhecido como Relatório Brundtland, inter-relaciona parâmetros como sustentabilidade, desenvolvimento e compromisso entre gerações atuais e futuras, sublinhando a interligação entre economia, tecnologia, sociedade e política; enquanto chama a atenção para uma nova postura ética, caracterizada pela responsabilidade tanto entre as gerações quanto entre os membros contemporâneos da sociedade atual (BRÜSEKE, 1993).

Neste sentido, assumem importância os cuidados que se referem à redução da extração de recursos naturais, bem como na minimização da disposição de resíduos. Ainda que a Química produza resíduos e tenha seus riscos inerentes aos processos, possui importante papel no desenvolvimento sustentável se aplicado os 12 princípios da Química Verde, visto que esta busca o avanço de tecnologias inovadoras para uma melhor qualidade de vida e um menor impacto ao meio ambiente, a exemplo da procura por processos incapazes de causar poluição e a diminuição de gastos com o armazenamento e tratamento de resíduos (PRADO, 2003).

É válido salientar que a Política Nacional de Resíduos Sólidos destaca a responsabilidade do gerador pelo gerenciamento dos resíduos (BRASIL, 2010, art. 10). No que diz respeito aos recursos hídricos há também a responsabilidade dos geradores de qualquer fonte poluidora em tratar para lançar os efluentes desde que estes obedeçam às condições, padrões e exigências dispostos na resolução $n^{\circ} 430$ do Conselho Nacional de Meio Ambiente, ou em outras normas que possam ser aplicadas (CONAMA, 2011).

Assim, as universidades, como polos de pesquisa com a função de ensino e extensão, devem auxiliar na difusão de tecnologias aplicadas à gestão de resíduos. Algumas universidades estaduais e federais, como a Universidade de São Paulo (USP), Universidade de Campinas (UNICAMP), Universidade Federal de Lavras (UFLA), Universidade Federal de São Carlos (UFSCAR), Universidade do Estado do Rio de Janeiro (UERJ), entre outras, já implantaram programas de gestão e de gerenciamento de resíduos químicos. Na USP, por exemplo, foi implantado o programa de gerenciamento de resíduos químicos no Laboratório de Isótopos Estáveis do CENA (Centro de Energia Nuclear na Agricultura), o qual economizou cerca de $\mathrm{R} \$ 72 \mathrm{mil}$ em 2003. O resultado positivo foi obtido aplicando-se a seguinte logística: prevenir, minimizar, reaproveitar, tratar, dispor. Segundo os autores, essa escala de prioridades, quando aplicada, permite resultados precisos e exatos, além de proporcionar um menor consumo de reagentes e a diminuição na geração de resíduos (TAVARES, 2005). 
Para o gerenciamento de resíduos químicos em laboratórios de ensino e pesquisa, uma das propostas apontadas por Jardim (1998) é a possibilidade de realizar as técnicas em micro escala, a fim de reduzir o volume e consumo dos reagentes utilizados. Jardim (1998) destaca que para o gerenciamento de resíduos químicos de laboratório ter eficácia é de grande importância o envolvimento de toda a comunidade acadêmico-científica, além de ampla divulgação e treinamento.

Neste sentido, a Universidade Federal de Alfenas (UNIFAL-MG), pode contribuir com a difusão de tecnologia para a minimização, tratamento e recuperação de resíduos químicos, visto que possui laboratórios que geram estes resíduos, além de destacada atuação no sul de Minas Gerais, e cujo crescimento prevê para os próximos anos a implantação de pelo menos mais três cursos de graduação como potenciais geradores de resíduos químicos em laboratórios de ensino e pesquisa.

O curso de Química da UNIFAL, por exemplo, possui um significativo destaque no quesito pesquisa e ensino e vem estudando constantemente novos materiais e métodos para os fins desejados em diversos contextos, utilizando, consequentemente, reagentes químicos com uma grande diversidade de composição.

Mesmo apresentando baixo volume, se comparados a unidades industriais, esses resíduos não recebem um tratamento químico ou uma destinação final padrão, o que dificulta a disposição final. Deve-se ressaltar que nos laboratórios são gerados resíduos compostos por metais potencialmente tóxicos, solventes halogenados, soluções ácidas e alcalinas, gases tóxicos, entre outros, que são muitas vezes tão nocivos quanto os resíduos industriais e também possuem forte contribuição para a poluição ambiental, requerendo, portanto, um cuidado todo especial por parte dos profissionais, quanto ao manuseio, ao correto tratamento e ao descarte final (SANTOS, 2006).

Como destacado por Benigno (2010), as instituições de ensino e pesquisa são quase sempre consideradas insignificantes geradoras de resíduos pelo fato de produzirem um pequeno volume de produtos, o que pode levar à negligência quanto ao destino final adequado dos resíduos das atividades acadêmicas e ao descarte correto destes. Isso porque as políticas governamentais e as legislações de controle dos riscos ambientais e de regulamentação à destinação final dos diversos produtos nocivos normalmente estão centradas nos resíduos químicos gerados pelas indústrias, devido ao grande volume e quantidade.

É de extrema importância a busca de rotas sintéticas que gerem efluentes de baixa toxicidade ou que possam ser facilmente reaproveitados, reciclados ou neutralizados. Não apenas por razões ecológicas, mas também por razões econômicas, já que se sabe que a melhor rota sintética é aquela que não gera resíduo, o que normalmente é difícil de ocorrer.

Nessa perspectiva, o gerenciamento dos resíduos químicos, frutos de atividades de ensino e pesquisa, tem ocupado cada vez mais espaço no meio acadêmico 
brasileiro, podendo ser considerado como uma aplicação que se chama hoje de química verde, química limpa, química ambientalmente benigna ou, ainda, química autossustentável (LENARDÃO, 2003).

Pela filosofia da Química Verde procura-se na prática desenvolver produtos ou processos químicos que reduzam ou eliminem o uso e a geração de substâncias perigosas. Assim, o foco e estratégia impostos se baseiam na preservação do meio ambiente, adotando novas fontes de energia, reagentes de custos mais baixos, que apresentem menor toxicidade, além de reações alternativas que gerem uma quantidade mínima de rejeitos.

Neste contexto, o presente artigo tem como objetivo apresentar uma proposta para implantação e gestão de um laboratório no campus II, Santa Clara, visando o tratamento de resíduos químicos da UNIFAL-MG (Alfenas), demonstrando que a implantação do mesmo é necessária e viável economicamente, socialmente e ambientalmente, podendo motivar a aplicação deste para outras estruturas de mesmo porte. Foi realizado um diagnóstico dos resíduos gerados e a partir deste levantamento foi verificada a infraestrutura física necessária para a construção do laboratório em questão, além dos equipamentos, vidrarias, mão de obra e materiais em geral que fossem necessários para que o laboratório pudesse entrar em operação, expondo os custos e investimentos necessários à implantação e gestão.

\section{Referencial teórico}

A Política Nacional de Resíduos Sólidos define o resíduo como o material, substância, objeto ou bem descartado que for resultante das atividades humanas em sociedade, no estado sólido ou semissólido, bem como gases contidos em recipientes e líquidos cujas particularidades tornem inviável o seu lançamento na rede pública de esgotos ou em corpos d'água, ou exijam para isso soluções técnicas ou economicamente inviáveis em face da melhor tecnologia disponível para a disposição final adequada (BRASIL, 2010, art. $3^{0}$ ).

Apesar de a definição ser dada a resíduos sólidos, também pode ser aplicada a resíduos líquidos. Neste sentido, também se aplica a classificação feita pela Associação Brasileira de Normas Técnicas (ABNT) na norma NBR 10004 (ABNT, 2004) que separa os resíduos sólidos em três categorias:

- Classe I ou Resíduos perigosos: são resíduos que apresentam periculosidade ou características como: inflamabilidade, corrosividade, reatividade, toxicidade e patogenicidade, podendo, portanto, ocasionar risco à saúde e ao meio ambiente;

- Classe II A ou Resíduos não perigosos e não inertes: não se enquadram nas outras classes, podendo ter como características a biodegradabilidade, a combustibilidade e a solubilidade em água. Nesta classe podem se enquadrar a 
maioria dos resíduos domésticos, sucatas de materiais ferrosos e/ou não ferrosos e embalagens de plásticos;

- Classe II B ou Resíduos não perigosos e inertes: são aqueles que através de uma amostra representativa e quando submetidos a um contato dinâmico e estático com água destilada ou deionizada, à temperatura ambiente, mantém todos seus constituintes não solubilizados.

Deve-se salientar que a Política Nacional de Meio Ambiente estabelece como um de seus objetivos a imposição ao poluidor e ao predador da obrigação de recuperar e/ou indenizar os danos causados (BRASIL, 1981, art.4 $4^{\circ}$ ). Portanto, torna-se de suma importância o gerenciamento dos resíduos visando à eliminação da geração, e não sendo isso possível, para os que fossem gerados, buscar a minimização na fonte ou por meio da reciclagem e do reuso, para dessa forma reduzir a destinação para tratamento e disposição final.

Qualquer ação de gerenciamento de resíduos requer a elaboração de um Programa de Gerenciamento de Resíduos (PGR) que deve incluir todas as atividades e os aspectos referentes à geração, segregação, armazenamento, coleta, transporte, tratamento até a disposição final do rejeito (PAIM; PALMA; EIFLER-LIMA, 2002).

Para que o Programa de Gerenciamento de Resíduos seja eficaz, é necessário considerar dois tipos de resíduos: o ativo, que é o principal alvo, fruto das atividades rotineiras da unidade geradora, e o passivo que corresponde ao resíduo não caracterizado, estocado, que estará aguardando a destinação final adequada (BENIGNO et al., 2010).

O gerenciamento de resíduos tem sido um tema bastante pertinente debatido com a comunidade acadêmica, que se preocupa com o desenvolvimento de atividades laboratoriais de ensino e pesquisa, com relação à geração e tratamento de resíduos frente à preservação ambiental (JARDIM, 1998). Devido à ausência de fiscalização, muitas instituições de ensino superior ainda não apresentam um programa de gerenciamento de resíduos, e grande parte do descarte ocorre de maneira inapropriada ou então indiscriminada (IMBROISI et al., 2006).

Desde os anos 90 o gerenciamento de resíduos químicos em laboratórios de ensino e pesquisa vem sendo discutido no Brasil. Diversas ações vêm sendo desenvolvidas por várias Instituições de Ensino Superior (notadamente públicas), buscando aumentar a visibilidade da importância do gerenciamento de resíduos químicos, como também os problemas referentes à falta deste (BENIGNO et al., 2010).

Através de um estudo realizado na Universidade de Campinas, foi constatado que para cada aluno que se formava no curso de química, consequentemente era gerada uma média de onze quilos de resíduos químicos, através das atividades desenvolvidas nas aulas práticas (BENDASSOLLI et al., 2003). Considerando que 
outras universidades e escolas técnicas apresentam carga horária de aulas práticas parecidas, essa média acaba se repetindo e contribuindo para um grande problema ambiental (PACHECO; HEMAIS, 2000).

De forma geral, a maior quantidade de resíduos gerados pelos Laboratórios (80\%) é de solventes não clorados; ácidos; bases e solventes clorados. Os solventes clorados são gerados em menores quantidades, entretanto, são os mais preocupantes devido à maior toxicidade e resistência para degradação. Em menores quantidades são gerados também metais pesados em solução, óxidos, corantes e sais (TAVARES, 2005).

Entretanto, o tratamento e a recuperação dos resíduos químicos podem ir além das fronteiras universitárias, com a prestação de serviços a empresas. No caso do presente trabalho podem ser atendidas as regiões sul e sudoeste de Minas Gerais (

Figura 1). Além disso, a troca de conhecimentos entre estudantes e funcionários e a possibilidade de estágios nas empresas podem ser fatores importantes para o crescimento profissional. Deve-se ressaltar que de acordo com levantamento sobre resíduos sólidos gerados por indústrias em Minas Gerais em 2012, no sul de Minas há 30 municípios e 63 empresas inventariadas, sendo que predominam as de material de transporte, têxtil e material elétrico-eletrônico (FEAM, 2013). Considerando as Superintendências Regionais de Meio Ambiente e Desenvolvimento Sustentável (SUPRAM), a do sul de Minas fica atrás apenas da SUPRAM Central e da SUPRAM Alto São Francisco, com 172 e 92 empresas inventariadas, respectivamente (FEAM, 2013).

\section{Figura 1 - Região sul e sudoeste de Minas Gerais}

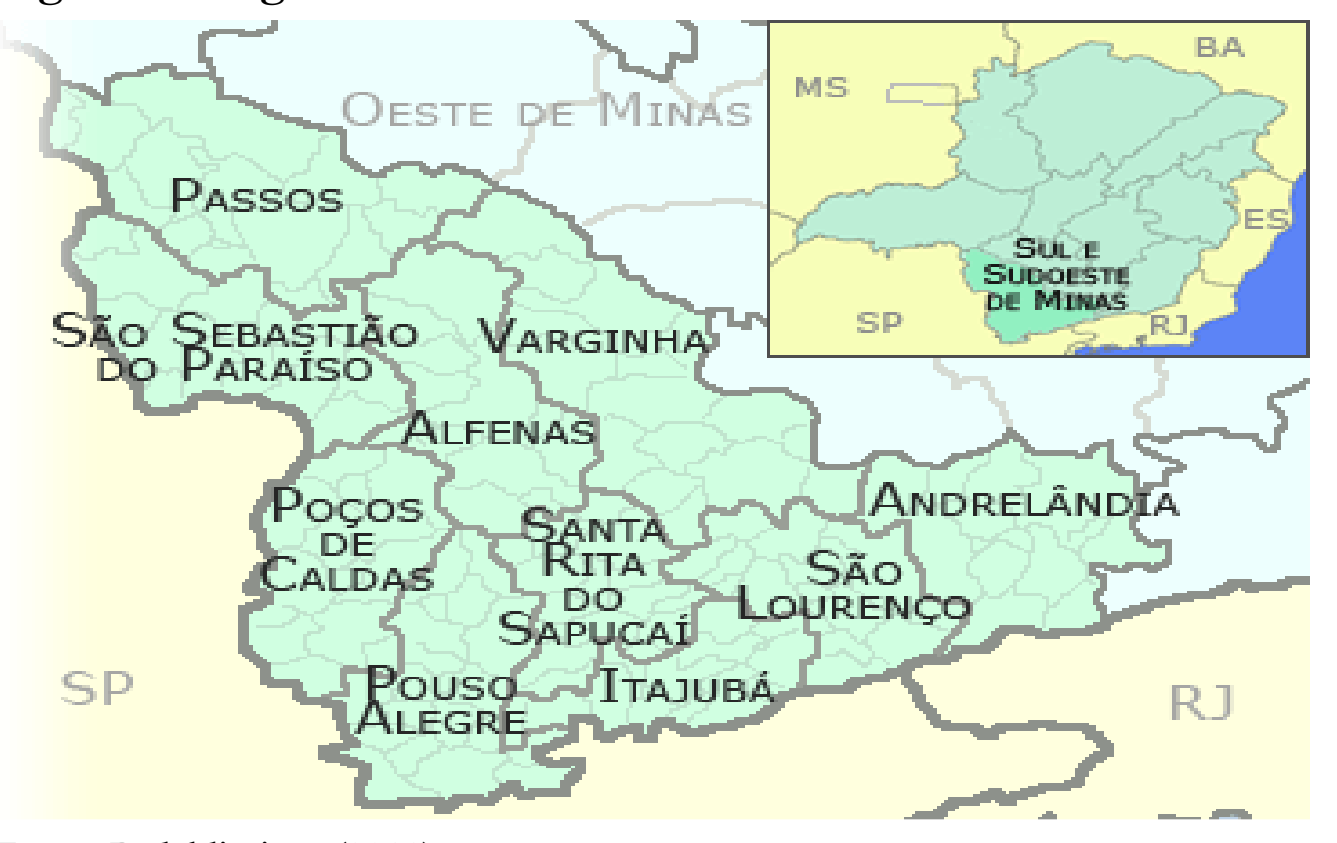

Fonte: Pedaldiminas (2013). 


\section{Material e métodos}

Para a quantificação dos resíduos químicos gerados pela Universidade foi elaborado um questionário (ver material suporte anexo) abordando os seguintes conteúdos: volume e tipos de resíduos gerados, forma de armazenamento e disposição final dos mesmos, tratamento e descarte. As entrevistas semiestruturadas foram respondidas por 18 docentes, 17 técnicos e 3 alunos de pós-doutorado ou mestrado, totalizando 38 entrevistados. Estes são usuários de 32 laboratórios de pesquisa, ensino e extensão. A etapa seguinte abrangeu um estudo sobre os recursos financeiros necessários para a construção e implantação de um Laboratório de Tratamento e Recuperação de Resíduos Químicos.

Todo investimento em um empreendimento, independente do porte, requer uma avaliação quantitativa e qualitativa sobre os recursos necessários para ser viabilizado. Para isto pode ser feito um estudo composto por uma análise criteriosa dos aspectos técnicos de produção e de desenvolvimento de um novo produto ou processo, bem como do seu potencial de mercado e de geração de retorno financeiro sobre o investimento realizado. Assim, deve ser feito um levantamento detalhado dos investimentos primários, como a previsão de custos com equipamentos, mão de obra, material de consumo, estrutura física, energia, água, dentre outros recursos, passando pela implantação até estabelecerem-se as operações e o advento do retorno de todo o processo. Desta forma, pode-se proporcionar ao investidor uma leitura prévia do negócio, dando-lhe maior segurança de modo a reduzir os riscos e as incertezas.

Portanto, neste trabalho o Estudo de Viabilidade Técnica e Econômica (EVTE) teve como objetivo verificar as condições para implantação do laboratório na UNIFAL-MG, e os parâmetros utilizados foram a Taxa Interna de Retorno (TIR) e o Valor Presente Líquido (VPL) seguindo as equações 1 e 2.

$\mathrm{VPL}=0=\sum_{\mathrm{t}=1}^{\mathrm{n}} \frac{(\text { Receita-Despesas }) \mathrm{t}}{(1+\mathrm{TIR})^{\mathrm{t}}}+$ investimentoinicial

$\mathrm{VPL}=\sum_{\mathrm{t}=1}^{\mathrm{n}} \frac{(\text { Receita-Despesas)t }}{(1+\mathrm{TIR})^{\mathrm{t}}}+$ investimentoinicial

O VPL é utilizado para a análise de fluxos de caixa e consiste no cálculo do valor presente de uma série de pagamentos, ou recebimentos, ou depósitos, a uma determinada taxa de juros e a TIR é definida como a taxa de juro composto (taxa de desconto) que anula o seu valor presente (valor atual). Ou, ainda, a taxa interna de retorno de um projeto é a taxa de juros para a qual o valor presente das receitas 
torna-se igual aos desembolsos. Pode ainda ser entendida como a taxa de remuneração do capital.

Diante do que foi apresentado, o projeto elaborado pode ser organizado com as seguintes etapas (Figura 2):

Figura 2 - Etapas do projeto realizadas (verde); Etapa que está sendo realizada (amarelo) e etapas futuras (vermelho).

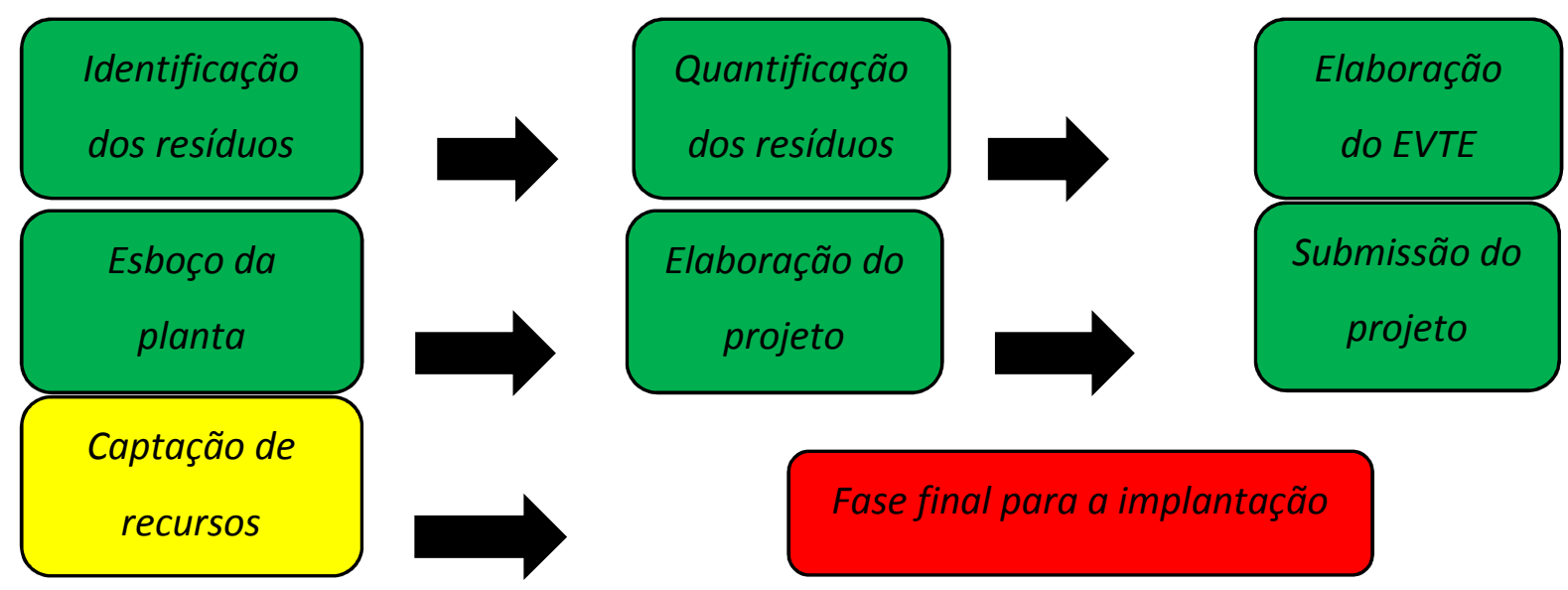

Fonte: Elaboração própria.

\section{Resultados e discussões}

Através da análise do questionário, pôde-se confirmar que na UNIFAL-MG, campus Alfenas, o volume total de resíduos químicos gerados pelos laboratórios é de aproximadamente $372 \mathrm{~L} /$ mês, somados a 3,5 kg/mês de sais inorgânicos.

A Figura 3 apresenta os resíduos gerados nos laboratórios. 
Figura 3 - Porcentagens de resíduos gerados, transformados de Kg, pelos laboratórios da UNIFAL-MG, campus Alfenas

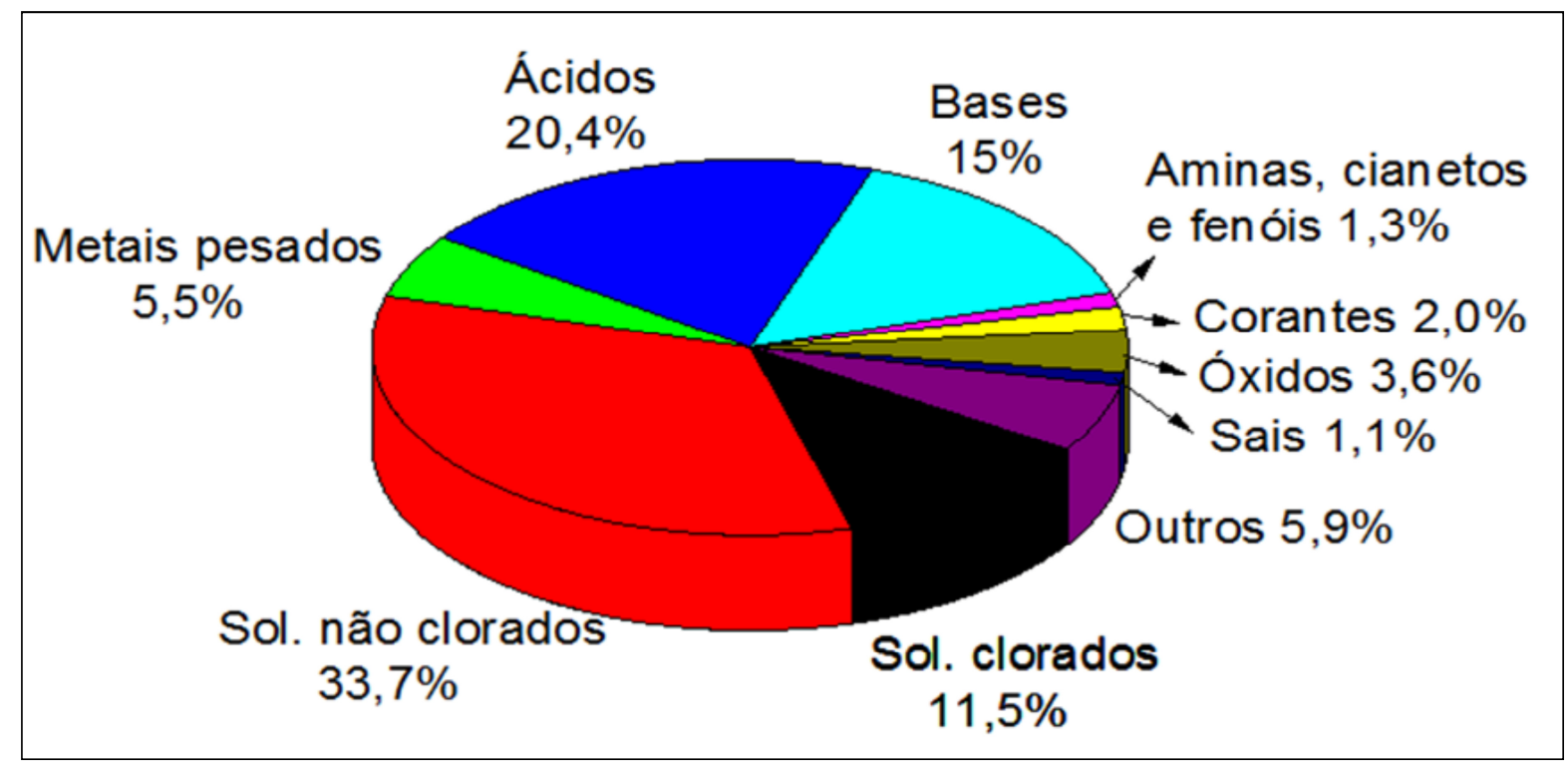

Fonte: Elaboração própria.

No questionário abordou-se também a opinião do entrevistado, entre docentes, técnicos e alunos, em relação à sua perspectiva de geração de resíduos para os próximos anos. A Figura 4 evidencia o resultado desta análise apontando que 50\% dos entrevistados acreditam que o processo de crescimento da UNIFAL-MG vai ocasionar o aumento da geração de resíduos.

Figura 4 - Previsão da geração de resíduos químicos pelos laboratórios da UNIFAL-MG para 2012 e 2013

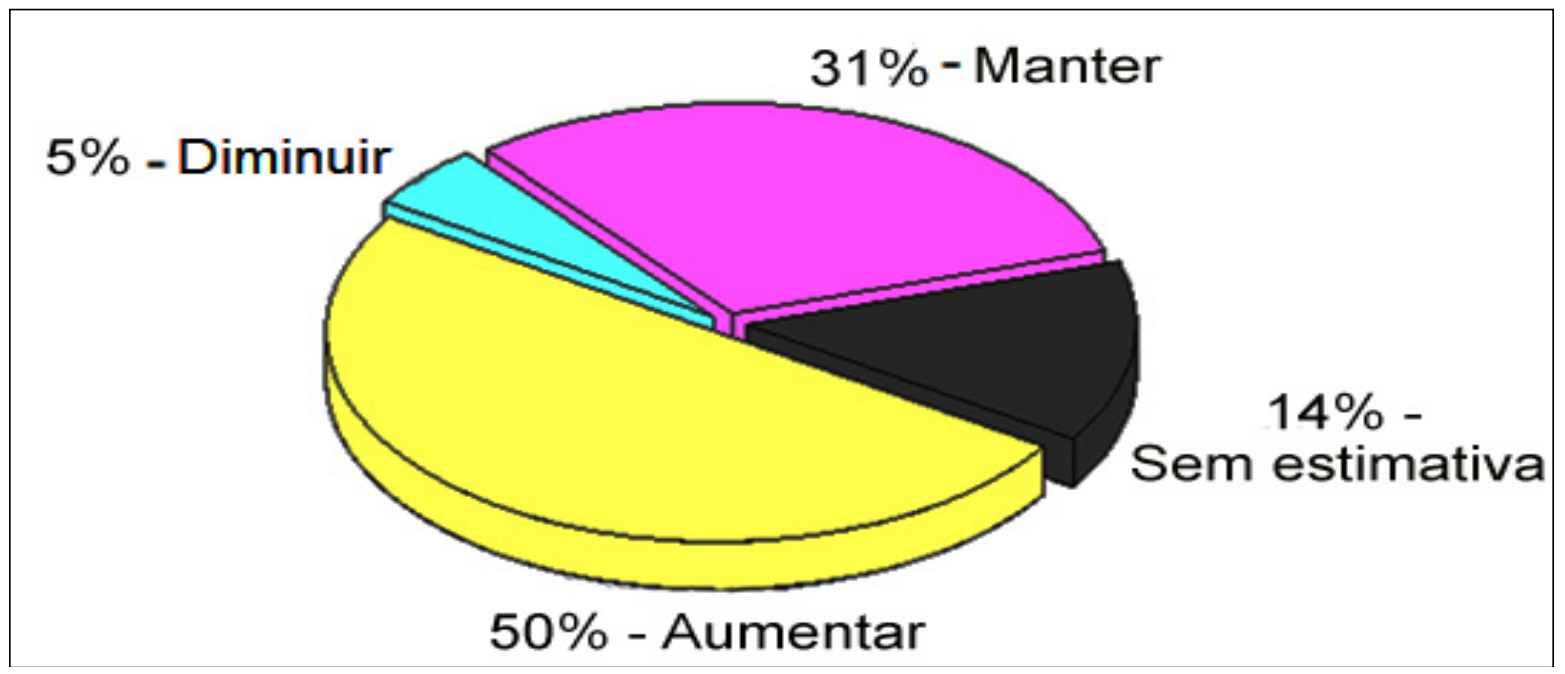

Fonte: Elaboração própria. 
Por outro lado, 31\% destes entrevistados acreditam que o volume de resíduos será mantido e outros 5\% esperam a redução deste volume. Assim, com base nos resultados da pesquisa, pode-se dizer que nos próximos anos a geração de resíduos irá aumentar consideravelmente em consequência da expansão da Universidade, visto que, os novos cursos que serão implantados, como medicina, utilizarão reagentes químicos. Isso reforça a necessidade da criação de um laboratório de tratamento e recuperação de resíduos químicos.

Fazendo uma análise financeira para a construção e compra de materiais para o laboratório torna-se indispensável a apresentação dos equipamentos necessários para o funcionamento do laboratório, como também os respectivos valores no mercado, segundo cotações realizadas em 2012 (Tabela 1). Para definir a quantidade e os equipamentos necessários para o tratamento e recuperação dos resíduos químicos foram considerados os dados obtidos no diagnóstico que estão representados nas Figuras 3 e 4. Vale ressaltar que o valor venal do terreno não foi levado em consideração pelo fato de se tratar de uma Instituição Pública Federal em que o terreno foi doado para a Universidade. Além disso, todos os impostos e taxas referentes à construção estão inseridos no valor da construção. Ainda referente à construção é importante salientar que o valor proposto foi definido com base nos valores médios firmados por licitação para $\mathrm{o} \mathrm{m}^{2}$ pela própria Universidade.

Tabela 1 - Orçamentos, em reais, de equipamentos e vidrarias para o laboratório de tratamento e recuperação de resíduos químicos

(continua)

\begin{tabular}{lccc}
\hline \multicolumn{1}{c}{ Equipamentos } & Quantidade & Preço previsto & Total \\
\hline Computadores & 2 & $\mathrm{R} \$ 1.000,00$ & $\mathrm{R} \$ 2.000,00$ \\
Capelas & 3 & $\mathrm{R} \$ 1.478,00$ & \\
& & $\mathrm{R} \$ 2.160,00$ & $\mathrm{R} \$ 7.435,00$ \\
Armários metálicos & 2 & $\mathrm{R} \$ 4.797,00$ & \\
Caixa de água & 3 & $\mathrm{R} \$ 142,00$ & $\mathrm{R} \$ 900,00$ \\
Estufa & 1 & $\mathrm{R} \$ 364,32$ & $\mathrm{R} \$ 649,52$ \\
Chapa aquecedora & 1 & $\mathrm{R} \$ 1.650,00$ & $\mathrm{R} \$ 1.650,00$ \\
Chuveiro e lava-olhos & 1 & $\mathrm{R} \$ 1.345,95$ & $\mathrm{R} \$ 1.227,88$ \\
Centrífuga (semi industrial) & 1 & $\mathrm{R} \$ 2.667,83$ & $\mathrm{R} \$ 2.667,83,95$ \\
Cruzeta horizontal & 1 & $\mathrm{R} \$ 471,00$ & $\mathrm{R} \$ 471,00$ \\
Balança semianalítica & 1 & $\mathrm{R} \$ 1.428,00$ & $\mathrm{R} \$ 1.428,00$
\end{tabular}


(conclusão)

\begin{tabular}{lccc} 
Balança analítica & 1 & $\mathrm{R} \$ 4.200,00$ & $\mathrm{R} \$ 4.200,00$ \\
Destilador & 5 & $\mathrm{R} \$ 1.750,00$ & $\mathrm{R} \$ 8.750,00$ \\
Espectrofotômetro & 1 & $\mathrm{R} \$ 6.896,47$ & $\mathrm{R} \$ 6.896,47$ \\
Autoclave & 1 & $\mathrm{R} \$ 3.360,50$ & $\mathrm{R} \$ 3.360,50$ \\
Phmetro de bancada & 1 & $\mathrm{R} \$ 495,00$ & $\mathrm{R} \$ 495,00$ \\
Bomba de vácuo & 2 & $\mathrm{R} \$ 1.101,10$ & $\mathrm{R} \$ 2.202,20$ \\
Forno mufla & 1 & $\mathrm{R} \$ 3.164,10$ & $\mathrm{R} \$ 3.164,10$ \\
Balança (p/ 300 kg) & 1 & $\mathrm{R} \$ 940,00$ & $\mathrm{R} \$ 940,00$ \\
Centrífuga $(100 \mathrm{~L} / \mathrm{h})$ & 1 & $\mathrm{R} \$ 980,00$ & $\mathrm{R} \$ 980,00$ \\
Vidraria e materiais em geral & - & $\mathrm{R} \$ 5.000,00$ & $\mathrm{R} \$ 5.000,00$ \\
\hline Total & $\mathbf{3 0}$ itens & - & $\mathbf{R} \$ \mathbf{5 5 . 7 3 3 , 4 5}$ \\
\hline
\end{tabular}

A Tabela 2 apresenta os investimentos financeiros necessários para construção e implantação do laboratório.

Tabela 2 - Investimentos estimados para implantação do laboratório

\begin{tabular}{lr}
\hline \multicolumn{1}{c}{ Investimentos } & $\mathrm{R} \$$ no $1^{\circ}$ ano (estimativa) \\
\hline Equipamentos & $50.733,45$ \\
Vidrarias e materiais & $5.000,00$ \\
Profissionais & $66.000,00$ \\
Construção (ver planta arquitetônica & $589.833,46$ \\
no material suplementar) & $\mathbf{7 1 1 . 5 6 6 , 9 1}$ \\
\hline Total & \\
\hline
\end{tabular}

Pode-se notar que serão necessários cerca de $\mathrm{R} \$ 645.566,91$ para a construção do laboratório e compra de equipamentos/vidrarias essenciais para seu funcionamento. Também serão necessários $\mathrm{R} \$ 66$ mil por ano para suprir as despesas com um químico e um técnico que serão responsáveis pelas atividades desenvolvidas no laboratório. Ressalta-se que o laboratório de tratamento e recuperação de resíduos químicos terá um docente como diretor, o qual será o responsável pela sua gestão.

Deve ser ressaltado que também podem ser previstas receitas a serem geradas pelo mesmo, as quais serão obtidas através da reutilização dos resíduos tratados, 
redução dos rejeitos enviados para empresas terceirizadas, prestação de serviços, expansão do patrimônio universitário, além da ampliação do espaço físico que poderá ser utilizado para o desenvolvimento de projetos de pesquisa por alunos de iniciação científica, mestrado, doutorado e pós doutorado, sem considerar aqui possíveis tecnologias e inovações que podem ser geradas das atividades deste Laboratório.

Por meio dos cálculos da TIR e do VPL (considerando uma inflação média anual de 5\% ao ano) foi possível estimar a viabilidade do projeto a ser realizado (Quadro 1).

\section{Quadro 1 - Cálculos da TIR e do VPL estimados}

\begin{tabular}{|c|c|c|}
\hline Período (anos) & 10 & \multirow{2}{*}{ Análise dos resultados } \\
\hline TIR estimada & $5,06 \%$ & \\
\hline VPL estimada & $\mathrm{R} \$ 1.615,40$ & $\begin{array}{l}\text { O Projeto é VIÁVEL para uma receita } \\
\text { média mínima anual de } \mathrm{R} \$ 170.000,00 \text {. }\end{array}$ \\
\hline
\end{tabular}

Portanto, a viabilidade econômica do projeto está ligada à redução de custos e despesas da universidade com tratamento de resíduos, visto que é contratada uma empresa terceirizada para esta finalidade, além da redução de aquisição de reagentes, já que estes poderão ser recuperados, bem como a possibilidade de prestação de serviço para toda região e aumento do patrimônio da UNIFAL-MG com a implantação de um novo laboratório.

Atualmente os resíduos químicos são coletados semanalmente por uma empresa terceirizada, a qual é responsável pela destinação, tratamento e disposição final, sob regime licitatório, que é oneroso aos gastos públicos, além das dificuldades peculiares ao processo, como tempo despendido entre a publicação do edital licitatório e a execução contratual. Deve-se considerar também o risco associado pela necessidade de assumir o princípio da corresponsabilidade, como previsto pela PNRS.

Assim, o custo gerado pode ser reduzido ou até mesmo transformado em receita, uma vez que o tratamento e recuperação dos resíduos químicos seriam realizados pela própria universidade. Este tratamento reduziria a disposição de resíduos químicos no meio ambiente, reduzindo os riscos de contaminação de áreas e consequentemente da biota e população local, e parte destes poderia ser recuperado e reutilizado em laboratórios de ensino para a realização de aulas experimentais contribuindo para a formação do aluno, o que implica em viabilidade ambiental e também social do projeto. Esta prática de tratamento e 
reutilização reduziria os custos institucionais com a compra de produtos químicos, o que tornaria possível a utilização de recursos para outros fins.

Neste âmbito, é indispensável ressaltar a Instrução Normativa $\mathrm{n}^{\circ} 10$, de 12 de novembro de 2012, a qual em seu Capítulo I, Art.1 ${ }^{\circ}$, institui regras para elaboração de Planos de Logística Sustentável na Administração Pública Federal. Entre os critérios da normativa, pode-se citar o Capítulo I, Art. $2^{\circ}$, contendo:

Parágrafo I - logística sustentável, processo de coordenação do fluxo de materiais, de serviços e de informações, do fornecimento ao desfazimento, que considera a proteção ambiental, a justiça social e o desenvolvimento econômico equilibrado;

Parágrafo II - critérios de sustentabilidade, parâmetros utilizados para avaliação e comparação de bens, materiais ou serviços em função do seu impacto ambiental, social e econômico;

Parágrafo III - práticas de sustentabilidade, ações que tenham como objetivo a construção de um novo modelo de cultura institucional visando a inserção de critérios de sustentabilidade nas atividades da Administração Pública.

E, seguindo este mesmo princípio, citando o Capítulo II, Art.8 ${ }^{\circ}$, Seção II, referente a práticas de sustentabilidade e racionalização do uso de materiais e serviços, pode-se ressaltar:

\footnotetext{
Parágrafo III - água e esgoto;

Parágrafo IV - coleta seletiva;

Parágrafo VI - compras e contratações sustentáveis, compreendendo, pelo menos, obras, equipamentos, serviços de vigilância, de limpeza, de telefonia, de processamento de dados, de apoio administrativo e de manutenção predial.
}

Deve-se ressaltar ainda que o tratamento e reutilização dos resíduos vão ao encontro do processo de educação dos alunos, visto que, como bem destacado por Pacheco e Hemais (2000), cumpre a função social da universidade e promove a conscientização de seus alunos sobre o meio ambiente, educando-os para que levem suas experiências como legado profissional, o que também corrobora a viabilidade social do projeto. Entretanto, como ressaltado por Jardim (1998) é de grande importância o envolvimento de toda a comunidade acadêmico-científica, ampla divulgação e treinamento para que o gerenciamento de resíduos químicos de laboratório tenha eficácia. 
Dessa forma, constatamos que o projeto é viável desde que a Universidade consiga tratar seus resíduos, prestar serviço para as empresas da região e diminuir os gastos gerados com compra de novos reagentes por esses estarem tratados, sem levar ainda em consideração o benefício no ensino, ambiental.

\section{Considerações finais}

Verificou-se neste trabalho que a proposta para implantação e gestão de um laboratório no campus II, Santa Clara, da UNIFAL-MG (Alfenas) para tratamento de resíduos químicos da Universidade atenderia a um volume inicial de aproximadamente $372 \mathrm{~L} /$ mês de resíduos químicos gerados pelos laboratórios somados a 3,5 kg/mês de sais inorgânicos, além de outros resíduos de indústrias da região. No primeiro ano seria necessário um investimento de $\mathrm{R} \$ \mathbf{6 4 5 . 5 6 6 , 9 1 \mathrm { em }}$ equipamentos, vidrarias, materiais e construção, além de $\mathrm{R} \$ 66$ mil por ano para os profissionais habilitados a gerir as atividades desenvolvidas no laboratório.

A construção e manutenção deste laboratório são viáveis economicamente dependendo do volume de resíduos tratados, recuperados ou reciclados, bem como da prestação de serviço para geradores de resíduos químicos da região. Assim, o custo de aquisição de novos produtos que foi evitado e a receita pela prestação de serviços deve corresponder a uma receita média mínima anual de $\mathrm{R} \$ 170$ mil.

A contratação de uma empresa terceirizada para a coleta dos resíduos químicos, quando comparada às atividades que serão realizadas no laboratório, mostra que a implantação deste é viável ambientalmente e socialmente pelo fato de reciclar, recuperar e reutilizar os reagentes químicos, sem a necessidade de extração de novos recursos. Isto também minimiza a disposição de resíduos químicos no meio ambiente, reduzindo os riscos de contaminação de áreas. Além disso, colabora para a formação acadêmica dos alunos, oferecendo oportunidades de pesquisas e estágios. Concomitantemente, a implantação do laboratório elimina a necessidade de processos licitatórios para tratamento e encaminhamento dos resíduos gerados na universidade, além da eliminação do risco assumido do princípio da corresponsabilidade; além de que atende às necessidades impostas por alguns dos princípios do Plano de Logística Sustentável na Administração Pública Federal.

\section{Referências}

BENDASSOLLI, J. A. et al. Gerenciamento de resíduos químicos e águas servidas no laboratório de isótopos estáveis do CENA/USP. Química Nova, 26, 612-617, 2003. 
BENIGNO, A. P. A. et al. Programa de gerenciamento de resíduos químicos no laboratório de química analítica de IQB/UFAL: uma aplicação da química verde. Revista Científica do IFAL, n. 1, v. 1. 2010.

BRASIL. Lei n. 9.433 de 08 de janeiro de 1997. Institui a Política Nacional de Recursos Hídricos, cria o Sistema Nacional de Gerenciamento de Recursos Hídricos, regulamenta o inciso XIX do art. 21 da Constituição Federal, e altera o art. $1^{\circ}$ da Lei $\mathrm{n}^{\mathrm{o}}$ 8.001, de 13 de março de 1990. Disponível em: <http://www.planalto.gov.br/ccivil_03/leis/L9433.htm>. Acesso em: 15 fev. 2014.

Lei n. 6.938 de 31 de agosto de 1981. Dispõe sobre a Política Nacional do Meio Ambiente. Disponível em: <http://www.planalto.gov.br/ccivil_03/ leis/16938.htm>. Acesso em: 15 fev. 2014.

. Lei n. 12.305 de 02 de agosto de 2010. Institui a Política Nacional de Resíduos Sólidos. Altera a Lei no. 9605, de 12 de fevereiro de 1998, e dá outras providências. Disponível em: <http://www.planalto.gov.br/ccivil_03/leis/ 16938.htm>. Acesso em: 15 fev. 2014.

DEBIT. Consulta de indicadores econômicos, UFIR. Disponível em: <http://www.debit.com.br/index.php>. Acesso em: 19 mar. 2015.

ESCOBAR, H. Meio ambiente é preocupação para 94\% dos brasileiros entrevistados pelo Ibope. O Estado de São Paulo, 04/08/2012. Disponível em: $<$ http://www.estadao.com.br/noticias/impresso,meio-ambiente-e-preocupacaopara-94-dos-brasileiros-entrevistados-pelo-ibope-,868445,0.htm $>$. Acesso em: 19 out. 2013.

ESTY, D. C. et al. Environmental sustainability index: benchmarking national environmental stewardship. New Haven: Yale Center for Environmental Law \& Policy, 2005.

IISD. International Institute for Sustainable Development. Measurement and assessment: Dashboard of sustainability. 2007. Disponível em: <http://www.iisd.org/cgsdi/dashboard.asp>. Acesso em: 15 jul. 2014.

IMBROISI, D. et al. Gestão de resíduos químicos em universidades: Universidade de Brasília em foco. Química Nova, 29, p. 404-409, 2006.

JARDIM, W. F. Gerenciamento de resíduos químicos em laboratórios de ensino e pesquisa. Química Nova, 21(5), p. 671-673, 1998. 
PACHECO, E. V.; HEMAIS, C. A. Gerenciamento de resíduos líquidos de laboratório: a experiência do IMA/UFRJ. Revista de Química Industrial, 716, p. 26-30, 2000.

PAIM, C. P.; PALMA, E. C.; EIFLER-LIMA, V. L. Gerenciar resíduos químicos: uma necessidade. Caderno de Farmácia, v. 18, n. 1. p. 23-31, 2002.

PEDALDIMINAS. Região sul de Minas Gerais. Disponível em: $<$ http://pedaldiminas.com.br/regiao-sul-de-minas-gerais $>$. Acesso em: 25 set. 2013.

PRADO, A. G. S. Química verde: os desafios da química do novo milênio. Química Nova, v. 26, n. 5, p. 738-744, 2003.

PRESCOTT-MIEN, R. The wellbeing of nations: a country-by-country index of quality of life and the environment. Island Press, p.367, 2001.

ROZENFELD, H. et al. Gestão de desenvolvimento de produtos. Uma referência para a melhoria do processo. São Paulo: Editora Saraiva, 2006.

SOUZA, A. L. L. Meio Ambiente e desenvolvimento sustentável: uma reflexão crítica. Papers do NAEA, Belém, n. 45, 1994.

TAVARES, G. A.; BENDASSOLLI, J. A. Implantação de um programa de gerenciamento de resíduos químicos e águas servidas nos laboratórios de ensino e pesquisa do CENA/USP. Química Nova, v. 28, n. 4, p. 732-738, 2005. 
Endereço para correspondência:

Luciano Tavaresda Costa - ltcosta@id.uff.br Outeiro de São João Batista, s/n 24020-141 Rio de Janeiro/RJ, Brasil

Eduardo Gomes Salgado - egsalgado@gmail.com

Gabriel Monteiro da Silva, 700, Prédio D

37130-000 Alfenas/MG, Brasil

Dirlane de Fátima do Carmo - dirlanefc@gmail.com

Rua Passo da Pátria, 156, Bloco D, Sala 235

24210-240 Niterói/RJ, Brasil

MayraGuerra-mayra.guerra1@yahoo.com.br

Gabriel Monteiro da Silva, 700, Prédio D

37130-000 Alfenas/MG, Brasil

Marina Evangelista - magodoi.quimica@gmail.com

Gabriel Monteiro da Silva, 700, Prédio D

37130-000 Alfenas/MG, Brasil

Karla Silveira - karlasde@gmail.com

Gabriel Monteiro da Silva, 700, Prédio D

37130-000 Alfenas/MG, Brasil 